\title{
ESTRATÉGIA DE SIMPLIFICAÇÃO MOLECULAR NO PLANEJAMENTO RACIONAL DE FÁRMACOS: A DESCOBERTA DE NOVO AGENTE CARDIOATIVO
}

\author{
Eliezer J. Barreiro*
}

Departamento de Fármacos, Faculdade de Farmácia, Universidade Federal do Rio de Janeiro, Cidade Universitária, Ilha do Fundão, CP 68006, 21944-190 Rio de Janeiro - RJ

Recebido em 24/1/02; aceito em 17/4/02

\begin{abstract}
STRATEGY OF MOLECULAR SIMPLIFICATION IN RATIONAL DRUG DESIGN: THE DISCOVERY OF A NEW CARDIOACTIVE AGENT. In this article are described examples of the successful use of molecular simplification strategy in the discovery of new drugs from bioactive natural products and synthetic compounds. The discovery of a new cardiotonic derivative (37, 2-thienylidene-3,4-methylenedioxybenzoylhydrazine; LASSBio-294), efficiently synthesized from Brazilian natural product and structurally designed by molecular simplification of active pyridazinone compounds reported in the literature, is described. A brief description of the pharmacological profile of this new cardiotonic lead-compound, belonging to the $N$-acylhydrazone (NAH) class, is also reported herein.
\end{abstract}

Keywords: new cardiotonic derivative; bioactive $N$-acylhydrazone compound; LASSBio-294.

\section{INTRODUÇÃO}

O processo de descoberta de novos fármacos, atribuição da Química Medicinal, caracteriza-se por sua complexidade, fruto da multiplicidade de fatores que envolvem o planejamento molecular de novas estruturas capazes de apresentarem os efeitos farmacológicos desejados, com biodisponibilidade adequada ao seu emprego terapêutico, seguro e confortável ${ }^{1,2}$.

Em virtude desta multiplicidade de fatores que, além da biodisponibilidade, incluem a toxicidade e o metabolismo, o processo de planejamento racional de novos fármacos envolve vários bilhões de dólares e ocupa milhares de pesquisadores titulados, de diferentes áreas, em diversos laboratórios de pesquisa, tanto industriais como acadêmicos. Este processo representou um desafio insuperável durante muito tempo, começando a ser vencido há ca. 30 anos, pela descoberta da cimetidina $(1)^{3,4}$, primeiro fármaco capaz de prevenir e curar a úlcera péptica. Este fármaco foi planejado para atuar como antagonista seletivo de receptores histaminérgicos do sub-tipo 2 (H-2) e representou, à época de seu lançamento, inovação terapêutica singular ${ }^{4}$.<smiles>CN/C(=N\C#N)NCCSCc1nc[nH]c1C</smiles>

O impacto terapêutico da descoberta da cimetidina $(1)^{3}$ no tratamento da úlcera péptica despertou o interesse de outros laboratórios industriais farmacêuticos, que desenvolveram outros antagonistas $\mathrm{H}$ 2, tipicamente como fármacos "me too" da cimetidina (1, Tagamet $\left.{ }^{\circledR}\right)$, empregando como estratégia de modificação molecular o bioisosterismo clássico de anéis ${ }^{5,6}$, inter-alia, ranitidina $\left(2\right.$, Zantac $\left.^{\circledR}\right)$, famotidina (3, Pepcid ${ }^{\circledR}$ ) e nizatidina (4), entre outros ${ }^{7}$.

*e-mail: eliezer@ufrj.br; Laboratório de Avaliação e Síntese de Substâncias Bioativas - LASSBio, http//:www.farmacia.ufrj.br/lassbio.<smiles>CN/C(=C/[N+](=O)[O-])NCCSCc1ccc(CN(C)C)o1</smiles>

2<smiles>CN/C(=C\[N+](=O)[O-])NCCSCc1csc(N=C(C)C)n1</smiles><smiles>CN/C(=C\[N+](=O)[O-])NCCSCc1csc(CN(C)C)n1</smiles>

Recentemente, face aos impressionantes avanços científicotecnológicos observados em diversas áreas como a biologia estrutural, molecular e a química computacional, por exemplo, o planejamento racional de novos fármacos tornou-se uma realidade.

A Figura 1 ilustra algumas das disciplinas envolvidas com o processo do planejamento racional de fármacos, destacando as tecnologias emergentes, que representaram significativas conquistas científicas do final do século 20, e que influenciarão sobremaneira este processo neste novo século. Vale destacar a contribuição da química computacional ${ }^{8}$ e, mais recentemente, da química combinatória ${ }^{9}$ que, acoplada ao contínuo desenvolvimento de computadores cada vez mais eficientes, viabilizou bioensaios robotizados de vastas quimiotecas que, provavelmente, desenharão novos 
paradigmas para o processo de descoberta racional de novos fármacos. A conquista do genoma e o desenvolvimento do proteoma ${ }^{10}$, além da nanotecnologia, representam aspectos recentes do avanço tecnológico que, certamente, terão enorme impacto neste processo.

Na Figura 1 estão ilustrados também alguns fármacos que poderiam ser merecedores do título de "fármaco da década", considerando-se o impacto terapêutico que representaram à época de seus lançamentos, tanto pelo nível de inovação quanto pelo índice de benefício à saúde que trouxeram.

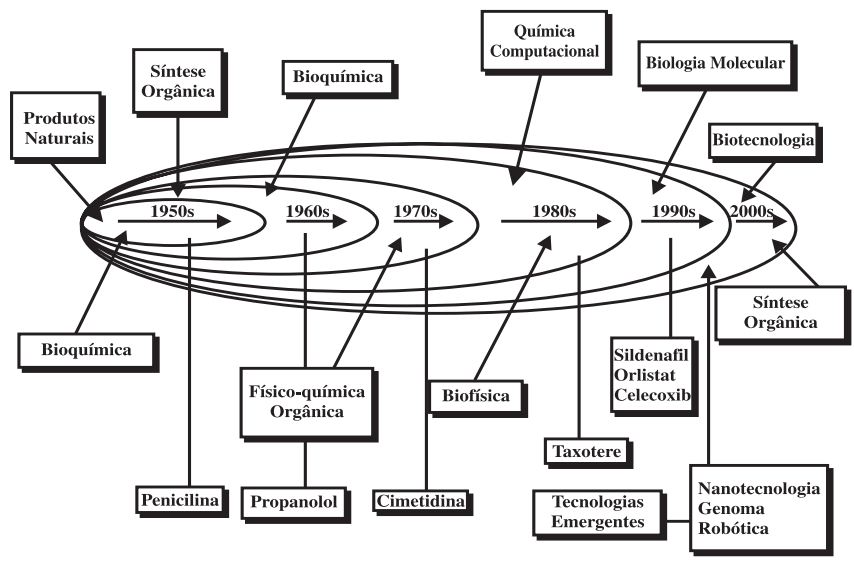

Figura 1. A interdisciplinaridade do processo da descoberta racional de fármacos

\section{A ABORDAGEM FISIOLÓGICA NA DESCOBERTA DE FÁRMACOS ${ }^{11}$}

A Química Medicinal dedica-se a estudar as razões moleculares da ação dos fármacos, a relação entre a estrutura química e a atividade biológica/farmacológica, além do planejamento racional de novos fármacos ${ }^{1,2}$. Em função da multiplicidade de fatores envolvidos na resposta terapêutica de uma substância exógena como o fármaco, o cumprimento desta difícil e complexa missão exige equipe interdisciplinar e fundamenta-se, simplificadamente, na tríade: Química Orgânica - Farmacologia - Química Computacional, ilustrada na Figura 2.

A eficácia que o fármaco deve apresentar depende de suas propriedades farmacodinâmicas e farmacocinéticas. As primeiras dependem das principais interações envolvidas no reconhecimento

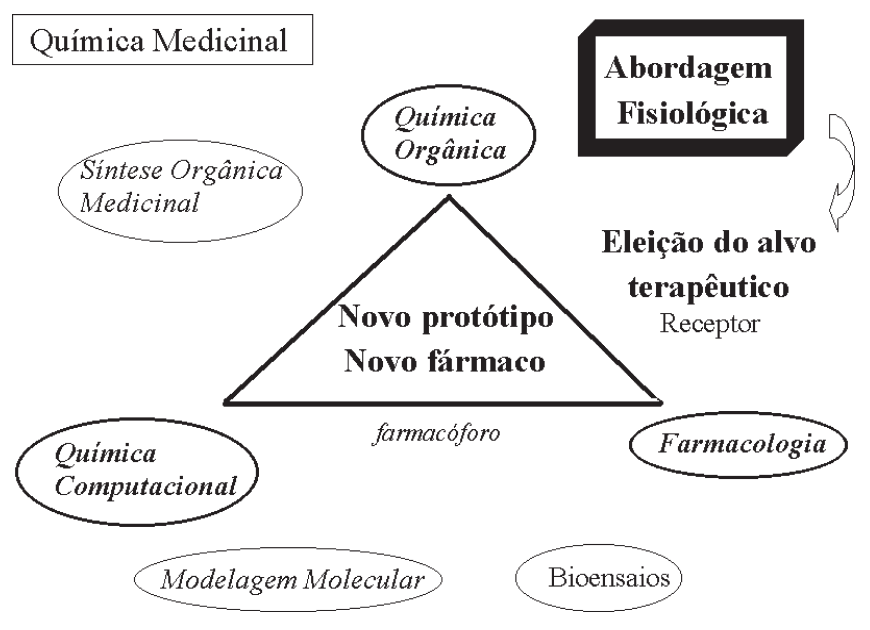

Figura 2. A tríade do processo de planejamento racional de fármacos baseada na abordagem fisiológica molecular dinâmico deste ligante pelo biorreceptor, i.e. grupos farmacofóricos, e definem sua afinidade. As últimas, governam os fatores de absorção, distribuição, metabolismo e eliminação do fármaco na biofase ${ }^{12}$. Este binômio definirá o perfil de eficácia e biodisponibilidade do fármaco.

Diversas estratégias de planejamento molecular de fármacos são disponíveis ${ }^{13}$, sendo aquela baseada no mecanismo de ação farmacológica pretendido, também denominada de abordagem fisiológica, a principal ${ }^{11,14}$.

A Figura 3, um detalhamento da Figura 2, ilustra, esquematicamente, as etapas hierárquicas da abordagem fisiológica ${ }^{11}$ para a descoberta racional de fármacos que subentende o prévio conhecimento da fisiopatologia em tela, condição essencial à correta eleição do alvo-terapêutico mais adequado, etapa inicial estratégica deste processo.

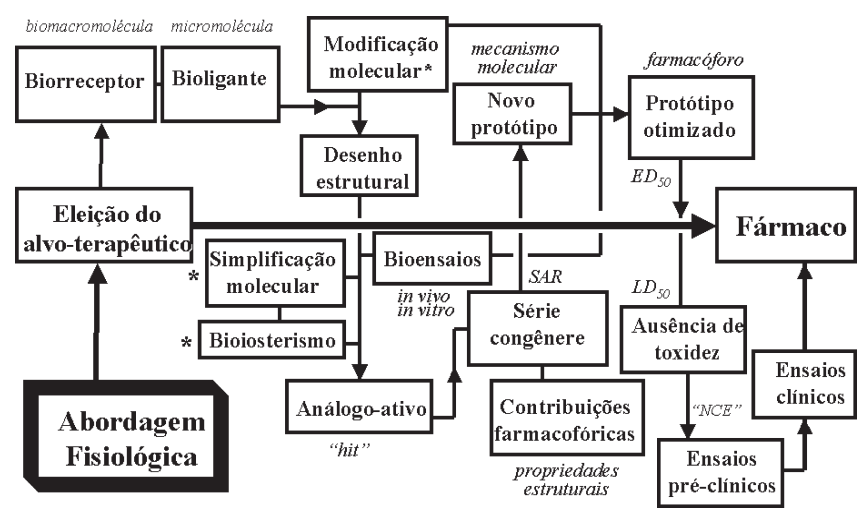

Figura 3. Etapas hierárquicas do processo de descoberta racional de fármacos baseado na abordagem fisiológica: da esquerda para direita, iniciando com a etapa crítica de eleição do alvo terapêutico $(S A R=$ relação estrutura-atividade; NCE = nova entidade química)

O alvo-terapêutico, representado por uma biomacromolécula enzima ou receptor, propriamente dito - pode ter sua estrutura molecular conhecida ou não (Figura 3$)^{14}$. Um terço dos fármacos atuais atuam como inibidores enzimáticos e cerca de 200 enzimas são alvos-terapêuticos, sendo que cerca de 9000 estruturas protéicas estão resolvidas por RMN ou raios-X e são disponíveis em bancos de dados. Em ambos os casos, o planejamento molecular de novo ligante/análogo-ativo pode ser realizado, seja partindo-se do substrato natural e introduzindo-se modificações moleculares, guiadas pelas estratégias clássicas da Química Medicinal, seja estudando-se os índices de complementaridade molecular entre bioligante-receptor com emprego de técnicas computacionais como a modelagem molecular ${ }^{15}$ e definindo-se os diferentes tipos de interações envolvidas ${ }^{14,15}$.

Entretanto, hèlas, nem sempre o bioligante ativo identificado in vitro neste processo racional apresenta perfil de biodisponibilidade adequado in vivo e, não raramente, novas modificações moleculares são necessárias para ajuste das propriedades farmacocinéticas ${ }^{16}$. Este ajuste será tão mais eficaz quanto se conheçam as contribuições farmacofóricas das diferentes sub-unidades estruturais do bioligante, o que permite preservá-las, assegurando, em tese, o reconhecimento molecular pelo biorreceptor.

Uma vez comprovada a atividade farmacológica desejada por meio de protocolos farmacológicos in vivo, tem-se um novo composto-protótipo (Figura 3), candidato a novo fármaco. Recentemente introduziu-se o termo "hit" para designar ligante ou análogo-ativo de baixa afinidade ou eficácia, respectivamente, representando uma etapa anterior à identificação do composto-protótipo.

O novo composto-protótipo pode ter sua eficácia otimizada por 
modificações estruturais subseqüentes (Figura 3), planejadas de forma a preservar as propriedades farmacocinéticas detectadas nos bioensaios in vivo. Uma vez otimizado o composto-protótipo identificado na série congênere bioativa e com comprovada ausência de toxicidade, tem-se uma nova entidade química ("NCE") ${ }^{17}$, estruturalmente original, que pode ser submetida às fases de ensaios préclínicos e clínicos, resultando, finalmente, num novo fármaco (Figura 3) ${ }^{14}$.

Para levar a termo e com sucesso esta missão, a Química Medicinal dispõe de diversas estratégias de planejamento molecular, dentre as quais a simplificação molecular de um protótipo-eleito. Neste processo, embora a intuição química do químico medicinal contribua enormemente, identificar e preservar as sub-unidades farmacofóricas é essencial ao sucesso do desenho dos novos padrões estruturais pretendidos.

Neste trabalho, descrevem-se exemplos do emprego desta estratégia na descoberta de fármacos originados de produtos naturais e compostos sintéticos bioativos e a descoberta de novo protótipo de fármaco cardioativo de origem sintética, da classe das $N$-acilidrazonas $(\mathrm{NAH})^{14}$, empregando produto natural abundante, como matéria-prima.

\section{A ESTRATÉGIA DE SIMPLIFICAÇÃO MOLECULAR}

A simplificação molecular foi inicialmente empregada na obtenção de compostos estruturalmente mais simples, a partir de protótipos naturais ativos, estruturalmente complexos. Esta estratégia de planejamento molecular de novos fármacos foi empregada em seus primórdios, empiricamente, sem o prévio conhecimento das diferentes contribuições farmacofóricas das distintas sub-unidades estruturais do protótipo, geralmente, mas não exclusivamente, de origem natural. Atualmente, os avanços observados no estudo da relação entre a estrutura química e a atividade permitiram que esta estratégia passasse a ser empregada de forma racional, preservando as sub-unidades farmacofóricas, previamente identificadas no composto-protótipo eleito, natural ou sintético ${ }^{18}$.

Em todos os processos de planejamento racional de novos bioligantes ou compostos-protótipos, deve-se considerar as eventuais contribuições toxicofóricas das sub-unidades estruturais destes protótipos ou bioligantes, principalmente ao nível do retículo microssomal hepático, responsável por oxidações dependentes do CYP450, essenciais à bioformação de substâncias endógenas e ao correto funcionamento de funções fisiológicas vitais ${ }^{12}$. A presença de grupos funcionais eletrofílicos (e.g. grupos funcionais aceptores de Michael, epóxidos) e sub-unidades estruturais extensamente coplanares devem ser consideradas como atributo de potencial hepatoe citotoxicidade, respectivamente, devendo ser, portanto, evitadas ${ }^{12}$.

\section{"Domesticando" produtos naturais bioativos}

Inúmeros alcalóides, termo introduzido em 1818 pelo químico alemão Wilhelm Meissner para denominar substâncias naturais básicas, de distintas classes, originaram importantes fármacos, de diversas categorias terapêuticas, pelo emprego, ainda empírico, à época, da estratégia de simplificação molecular do produto natural protótipo.

Um exemplo clássico é a morfina (5), alcalóide isolado de Papaver somniferum (Papaverácea) pelo farmacêutico alemão F. W. A. Setürner, em 1803. Este fármaco foi o precursor dos analgésicos da classe da 4fenilpiperidinas, representados pela petidina $(6)^{19}$, ilustrando significativamente o sucesso da estratégia de simplificação molecular, sucessiva, de protótipos naturais. A estrutura da petidina (6) caracterizase pela presença de um sistema piperidínico similar àquele contido na morfina (5), em que o carbono C-4, quaternário, encontra-se ligado ao anel benzênico (Esquema 1) ${ }^{19}$. Este correspondia ao grupo farmacofórico clássico dos hipnoanalgésicos opiáceos.

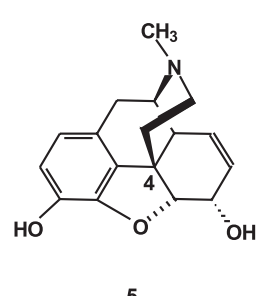

5

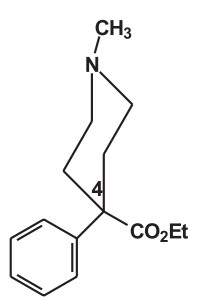

6

\section{Esquema 1}

A quinina (7), um dos principais componentes da casca de Cinchona officinalis, é outro exemplo clássico ${ }^{20}$. Este alcalóide originou, mais recentemente, por simplificação molecular planejada, os derivados antimaláricos da classe dos hidroximetilquinolínicos ou quinolinometanóis, exemplificados pela mefloquina $(8)^{21}$ (Esquema 2). O processo de simplificação molecular que originou a mefloquina (8) compreende, basicamente, a modificação do anel rubânico da quinina (7) para o sistema piperidínico. A presença dos substituintes triflúormetila nos carbonos 2 e 8 do sistema quinolínico foi ditada por razões metabólicas. A mefloquina (8) veio a suplantar os fármacos mais antigos das classes das 4-aminoquinolinas, exemplificado pela cloroquina (9, Esquema 6).

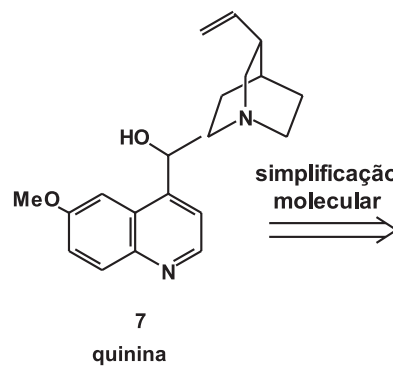<smiles>OC(c1cc(C(F)(F)F)nc2c(C(F)(F)F)cccc12)C1CCCCN1</smiles>

Esquema 2

O aciclovir (10, Zovirax $\left.{ }^{\circledR}\right)^{22}$, descoberto, em 1977, nos laboratórios Welcome, EUA, por Gertrude Elion e colaboradores, é um derivado purínico sintético, obtido por simplificação molecular ou abertura de anel (processo especial de modificação molecular) da guanosina (11) (Esquema 3) é empregado até hoje, com sucesso, no tratamento do Herpes sp..

O captopril (12, Capoten $\left.{ }^{\circledR}\right)$, descoberto em 1977, nos laboratórios Squibb, por Ondetti e colaboradores ${ }^{23}$, é um fármaco anti-

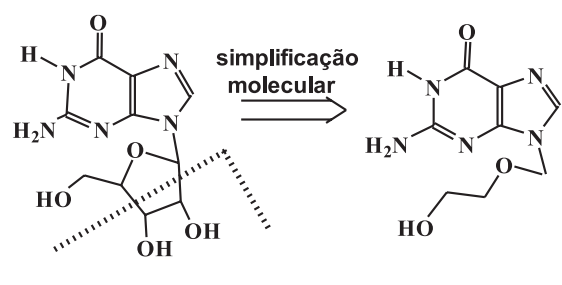

11

10 
hipertensivo, peptóide, desenvolvido por simplificação molecular consecutiva do nonapetídeo teprotido (13, SQ-20.881). Este último possui a mesma seqüência dos peptídeos isolados por Ferreira e colaboradores, em 1965, do veneno de jararaca (Bothrops jararaca) ${ }^{24}$, identificados como inibidores da enzima conversora de angiotensina (ECA). Esta enzima promove a hidrólise de ligações peptídicas envolvendo a prolina, o que explica a presença do sistema pirrolidônico em 12, que apresentou $\mathrm{IC}_{50}$ de $0,023 \mu \mathrm{M}$ com $K_{\mathrm{i}}$ de $0,0017 \mu \mathrm{M}$. Inibidores da ECA previnem a bioformação da angiotensina II, endopeptídeo pró-vasoconstritor e, simultaneamente, a degradação da bradicinina, potente agente hipotensor endógeno e representaram, à época, importante inovação terapêutica para o tratamento da hipertensão (Esquema 4).

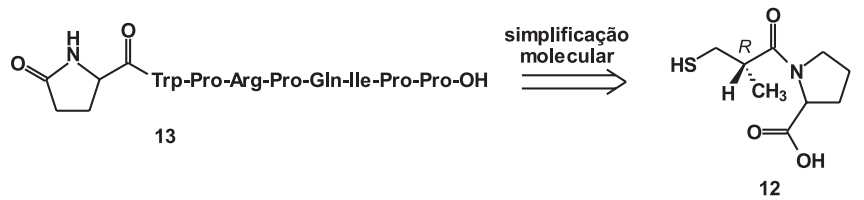

Esquema 4

\section{Fármacos sintéticos}

Dentre os fármacos antiinflamatórios não-esteróides (NSAI) que atuam como inibidores da enzima prostaglandina endoperóxido sintase (PGHS) ${ }^{25}$, descobertos pelo emprego da estratégia de simplificação molecular, encontra-se, entre outros, o zomepiraco (14) (Esquema 5$)^{26}$. Este agente NSAI foi estruturalmente planejado por simplificação molecular da indometacina (15), protótipo da classe dos agentes NSAI do grupo dos ácidos aril-acéticos descoberto por Shen e colaboradores, em $1962^{27}$. O planejamento estrutural do zomepiraco (14) foi inspirado na necessidade de se excluir o sistema indólico do protótipo, responsabilizado pelos efeitos adversos sobre o SNC provocado pela indometacina (15) e metabólitos em alguns pacientes (Esquema 6).

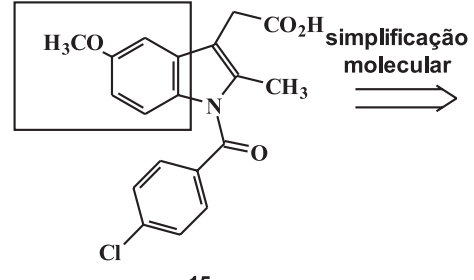

15

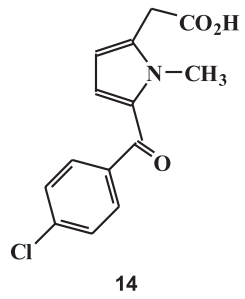

Esquema 5

No domínio dos quimioterápicos a cloroquina (9) é um exemplo clássico. Este fármaco antimalárico resultou do emprego da estratégia de simplificação molecular sobre a mepacrina (16), antimalárico da classe das 9-aminoacridinas, descrita em 1932 (Esquema 6).

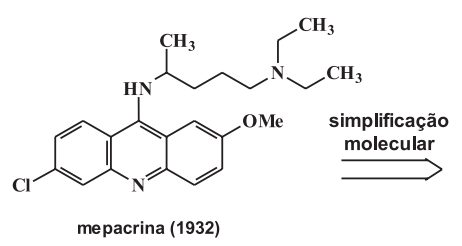

(16)

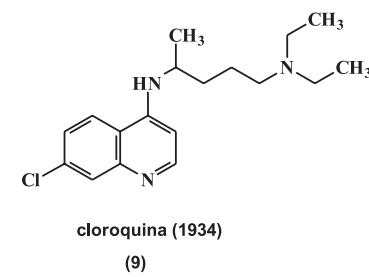

(9)
O indinavir (17, Crixivan $\left.{ }^{\circledR}\right)^{28}$, fármaco antiviral empregado no tratamento da síndrome da imunodeficiência adquirida (SIDA), atua como inibidor de protease viral, i.e. Asp-proteases (Esquema 7) ${ }^{29}$. Este agente antiviral contemporâneo é exemplo recente da descoberta de fármacos baseada na abordagem fisiológica, aplicando-se a estratégia de simplificação molecular de um protótipo desenvolvido a partir do conhecimento da estrutura 3D do sítio receptor. A partir da estrutura de Asp-proteases, o protótipo (18) foi planejado, representando a segunda geração de candidatos a inibidores desta enzima, essencial no ciclo evolutivo do vírus, compreendendo a otimização dos derivados ureídicos inicialmente identificados ${ }^{30}$. A substância (18) possui na sub-unidade estrutural $\mathbf{A}$, i.e. sistema cis-peridroisoquinolínico funcionalizado, três centros estereogênicos (Esquema 7). A simplificação molecular deste sistema por eliminação de dois destes centros e com a inclusão do anel isostérico piperazínico $N$-substituído originou o indinavir $(17)^{28}$.

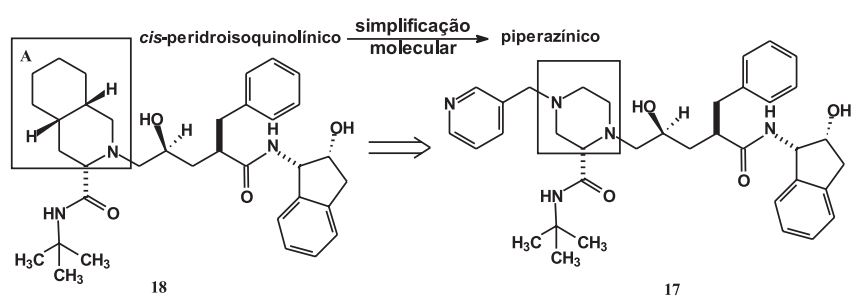

Esquema 7

\section{APLICAÇÃO DA ESTRATÉGIA DE SIMPLIFICAÇÃO MOLECULAR: A DESCOBERTA DE NOVO AGENTE CARDIOATIVO (LASSBIO-294)}

As fosfodiesterases (PDE's) são importantes classes de enzimas que promovem a hidrólise do monofosfato cíclico de adenosina e guanosina ( $c$ AMP e $c$ GMP) (Esquema 8$)^{31}$. Estes segundos mensageiros são responsáveis pela mediação de diversas respostas fisiológicas vitais, envolvendo diferentes hormônios, neurotransmissores ou autacóides. O aumento da taxa celular destes nucleotídeos cíclicos, por exemplo, promove a ativação de proteínas quinases (PK's) que, por sua vez, efetuam a fosforilação de diferentes tipos de substratos, regulando inúmeras respostas celulares ${ }^{31}$.

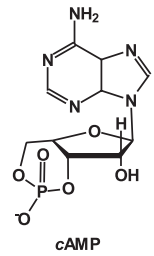

cAMP

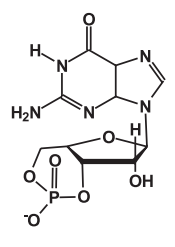

$c \mathrm{GMP}$

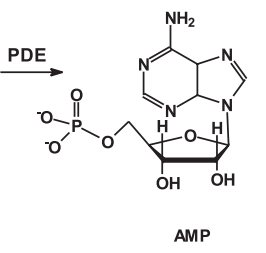

AMP

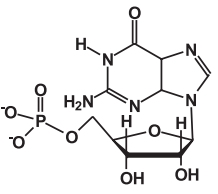

GMP
Esquema 8

A compreensão das bases bioquímicas deste processo fez com que as PDE's fossem reconhecidas como importantes alvosterapêuticos, despertando o interesse dos químicos medicinais ${ }^{31}$.

Atualmente são conhecidas $c a$. 11 isoformas de PDE's (PDE1PDE11) ${ }^{32,33}$, amplamente distribuídas em diferentes tecidos ou células como coração, cérebro, neutrófilos, linfócitos-T, macrófagos, eosinófilos, plaquetas, entre outros, classificadas em função do nucleotídeo cíclico que elegem como substrato específico ou preferencial ${ }^{31}$. O efeito de um inibidor seletivo de PDE's promove acúmulo de $c$ AMP ou $c$ GMP em determinado tecido, modulando, por exem- 
plo, canais iônicos celulares e alterando o estado de ativação destas células, resultando, por exemplo, no relaxamento da musculatura lisa ou na redução da atividade de células inflamatogênicas ${ }^{34}$.

A isoforma PDE4, específica para $c$ AMP, por exemplo, pode apresentar, ainda, sub-tipos (PDE4A-D) ${ }^{35}$ e é aquela predominantemente envolvida na resposta inflamatória, sendo inibidores seletivos desta isoforma fármacos como o rolipram (19), capazes de reduzirem a produção de citocinas flogísticas e atenuarem a evolução da resposta inflamatória do processo asmático ${ }^{36,37}$.

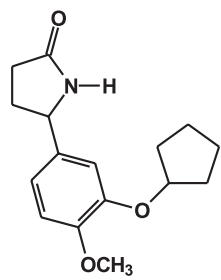

19<smiles>CCCc1nn(C)c2c(=O)[nH]c(-c3cc(S(=O)(=O)N4CCN(C)CC4)ccc3OCC)nc12</smiles>

20
Um exemplo recente do sucesso terapêutico do emprego de inibidores de PDE's é o sildenafil (20, Viagra $\left.{ }^{\circledR}\right)^{38}$, fármaco útil para o tratamento da disfunção eréctil, lançado em 2000 pelos laboratórios Pfizer e que representa importante inovação terapêutica, atuando como inibidor seletivo da PDE5.

\section{A descoberta de novo protótipo cardiotônico LASSBio-294} (37)

A fisiopatologia da insuficiência cardíaca caracteriza-se pelo infarto do miocárdio com edema e congestão periférica. A terapia geralmente empregada para o tratamento destes quadros inclui o uso de fármacos capazes de aumentarem a contratibilidade do miocárdio com efeitos inotrópicos positivos, e.g. glicosídeos cardíacos ${ }^{39}$, combinados com diuréticos. Infelizmente o uso dos glicosídeos cardiotônicos tem sido restrito, em função de seus efeitos próarritmogênicos e do reduzido índice terapêutico que possuem. Além destes cardiotônicos que parecem atuar sobre a $\mathrm{Na}^{+}-\mathrm{K}^{+}$-ATPase, fármacos inibidores da enzima conversora de angiotensina (ACE) $)^{40}$, antagonistas de receptores de angiotensina ${ }^{41}$, antagonistas de canais de cálcio ${ }^{42}$, e antagonistas adrenérgicos ${ }^{43}$ têm sido empregados no tratamento da insuficiência cardíaca.

$\mathrm{O}$ envolvimento do $\mathrm{Ca}^{+2}$ miofibrilar e da isoforma cardíaca de PDE3, que apresenta a mesma afinidade para ambos nucleotídeos $c$ AMP e $c$ GMP, tem antecipado a possibilidade de se tratar a insuficiência cardíaca congestiva com inibidores desta isoforma, capazes de promover efeitos combinados vasodilatadores e inotrópicos positivos $^{44}$.

Entre os inibidores conhecidos da PDE3 ${ }^{45}$ encontram-se diversos derivados piridazinônicos sintéticos ${ }^{46}$ (21 e 22, Esquema 9) ${ }^{47,48}$. Estas substâncias (21 e 22) originaram-se dos derivados piridazinônicos (23), estruturalmente relacionados à classe dos cardiotônicos 2-(1H)piridonas (24) a que pertence a milrinona $(25)^{49}$, fármaco cardiotônico, inibidor de PDE3, lançado em 1981 pelos laboratórios Sterling Co., EUA.

Dentre os compostos PDE-ativos da classe dos derivados piridazinônicos (23), a zardaverina (26) apresentou propriedades inibidoras seletivas para a isoforma de PDE $4^{50}$. Embora estruturalmente similar, o imazodam $(21)^{48}$, primeiro derivado diidro $(2 H)$ piridazinônico descrito, possui propriedades inibidoras da isoforma PDE3 e foi identificado como fármaco cardiotônico. O pimobendam $(22)^{48}$, derivado da mesma classe, descrito em 1982 como novo agente inotrópico positivo, também atua como inibidor de PDE3, diferindo
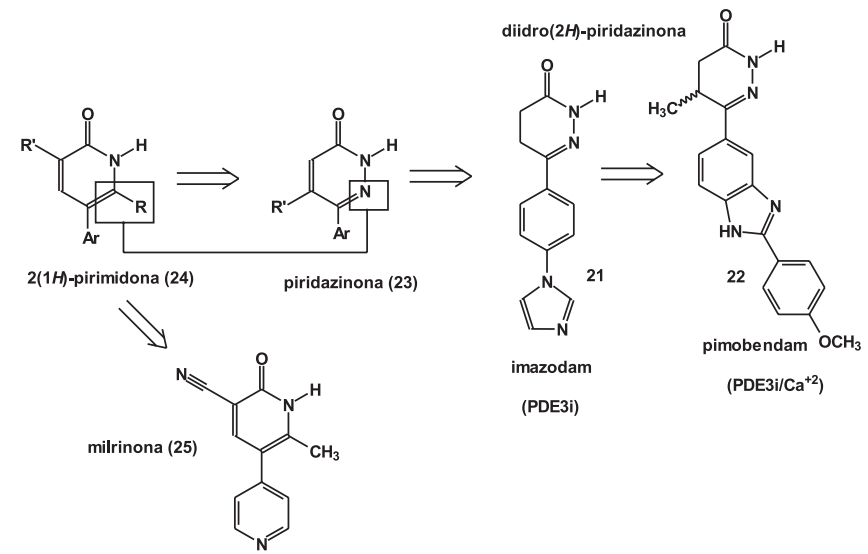

Esquema 9

de (21) por apresentar reduzidos efeitos pró-arritmogênicos ${ }^{48}$ e maior eficácia contrátil, devido à sua ação sobre o $\mathrm{Ca}^{+2}$ celular ${ }^{51}$.

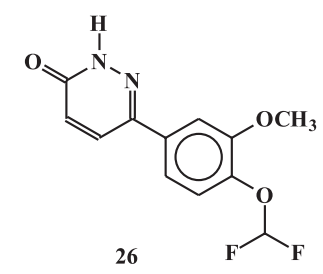

Há algum tempo vimos estudando no Laboratório de Avaliação e Síntese de Substâncias Bioativas (LASSBio) da Faculdade de Farmácia da Universidade Federal do Rio de Janeiro (UFRJ), o planejamento, a síntese e as propriedades farmacológicas de novos derivados $N$-acilidrazônicos (NAH) bioativos ${ }^{14}$, tendo sido identificados inúmeros compostos com atraentes propriedades antiflogísticas, antitrombóticas e analgésicas ${ }^{52-53}$.

O emprego da estratégia de simplificação molecular, ilustrada no Esquema 10, sobre os derivados diidro( $2 H)$ piridazinônicos $(21$ e 22), representada pela simples ruptura da ligação $\boldsymbol{a}$ permitiu identificar-se a função NAH, mascarada no sistema heterocíclico de (21) e (22).

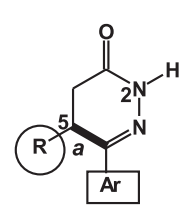

2H-piridazinona

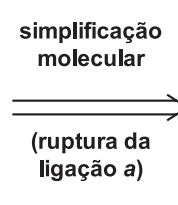

$$
N \text {-acilidrazona }
$$

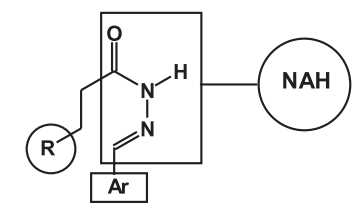

Esquema 10

A aplicação desta estratégia de simplificação molecular sobre os inibidores de PDE3 (21) e (22), possuindo importantes propriedades cardioativas, permitiu o reconhecimento da similaridade molecular existente entre derivados $N$-acilidrazônicos e estes compostos cardioativos, estimulando-nos ao planejamento de novos derivados $\mathrm{NAH}$ (27, Esquema 11). Estes foram investigados quanto às suas eventuais propriedades cardiotônicas, ampliando as perspectivas de emprego terapêutico desta classe de derivados de contínuo interesse do laboratório, tipicamente simples do ponto de vista estrutural e, portanto, de fácil acesso sintético, atributos fascinantes para o químico medicinal. 


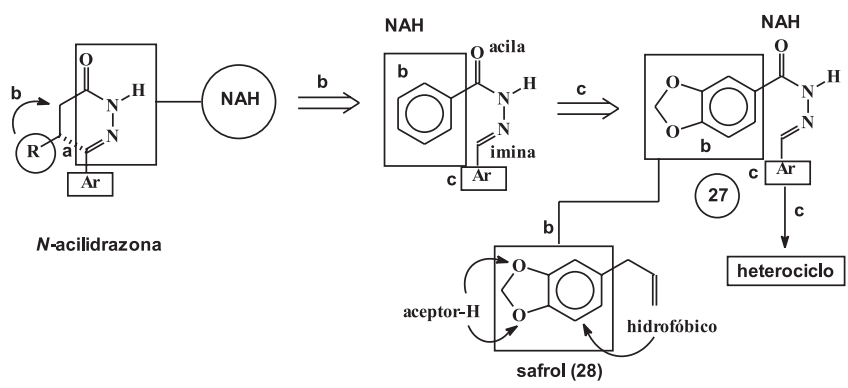

Esquema 11

A gênese da nova série de derivados NAH (27) (Esquema 11) fundamentou-se na inclusão de unidade aromática $\boldsymbol{b}$ na função acila, eliminando-se o carbono estereogênico C-5, presente em derivados (2H)-piridazinônicos ativos como o pimobendam (22) e outros (Esquema 10$)^{54}$. Existem relatos na literatura que descrevem a estereoespecificidade na ação dos enântiomeros desta classe de derivados sobre a PDE $3^{54}$.

A natureza da sub-unidade estrutural $\boldsymbol{b}$ incluída nos novos derivados NAH (27) inspirou-se no safrol (28) (Esquema 11), principal componente químico do óleo de Sassafrás e amplamente estudado em nosso laboratório como matéria-prima para a síntese de substâncias bioativas ${ }^{55}$. O reconhecimento do caráter ambidente do sistema benzodioxol presente em (28) decorre da propriedade em interagir com possíveis sítios de reconhecimento molecular de receptores biológicos atuando como aceptor de ligações-H e por interações hidrofóbicas (Esquema 11), simultaneamente, o que o credencia como um atraente bióforo, como comprovam resultados anteriores obtidos no laboratório ${ }^{55}$.

Uma vez definido o padrão estrutural da sub-unidade $\boldsymbol{b}$ dos novos derivados NAH planejados (27), restava-nos eleger o padrão estrutural do substituinte aromático $c$ (Esquema 10) da insaturação iminíca de (27).

O dantroleno (29, Esquema 12), fármaco descrito em $1986^{56} \mathrm{e}$ introduzido na terapêutica como agente miorrelaxante esquelético, atuando, seletivamente, na liberação do $\mathrm{Ca}^{+2}$ do retículo sarcoplasmático (SR), é o único fármaco efetivamente indicado no tratamento da hipertermia maligna ou da esclerose múltipla ${ }^{57} \mathrm{e}$, recentemente, seu mecanismo de ação foi elucidado envolvendo os canais de $\mathrm{Ca}^{+2}$ modulados pelos receptores de rianodina ${ }^{58}$.

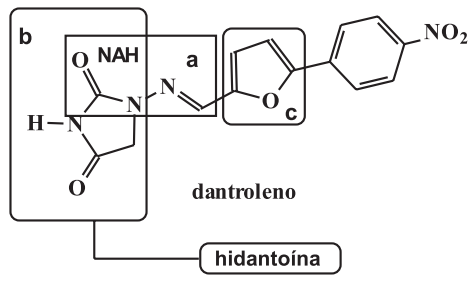

29

Esquema 12

A análise da estrutura deste fármaco hidantoínico (29) evidencia, mais uma vez, a presença da função NAH ( $\boldsymbol{a}$, Esquema 12) parcialmente incluída no anel imidazolinodiônico (b, Esquema 12) e substituída na insaturação imínica pelo núcleo 2-furano-5funcionalizado (c, Esquema 12). Esta análise estrutural inspirou a natureza heterocíclica do padrão de substituição aromática $\boldsymbol{c}$ (Esquema 11) da insaturação $\mathbf{C}=\mathbf{N}$ da nova classe de derivados NAH planejados (27)(Esquema 11) ${ }^{14,52}$.
O Esquema 13 ilustra a rota sintética empregada para a preparação dos novos derivados NAH (27) que se fundamentou no emprego de metodologia clássica, amplamente utilizada na síntese de inúmeras e distintas séries de derivados NAH bioativos, descritos por Barreiro e colaboradores ${ }^{14,52,59}$. Preparação do éster (30) (Esquema $13)^{60}$, a partir do safrol (28), por isomerização inicial da insaturação presente no produto natural ${ }^{61,62}$, levou ao isômero estirênico (31) em $80 \%$ de rendimento ${ }^{52,61}$. Subseqüente ozonólise do isosafrol (31) produziu, em bons rendimentos, o aldeído (32) ${ }^{61}$. Oxidação de (32) pelo método de Yamada (iodo, hidróxido de potássio em metanol) ${ }^{63}$ forneceu o éster desejado (30), em $68 \%$ de rendimento global médio a partir do safrol (28). Hidrazinólise do éster de (30) produziu a hidrazida correspondente (33) que, por condensação ácido- catalisada com os diversos heteroaril-aldeídos, forneceu os derivados NAH desejados $(27)^{52}$. O uso do 2-furfural (34) ou 2-formiltiofeno $(35)^{64}$ na etapa de condensação ácido-catalisada produziu os novos derivados (36) e (37), em ca. 62\% de rendimento global a partir do éster (30) (Esquema 13) ${ }^{64}$.

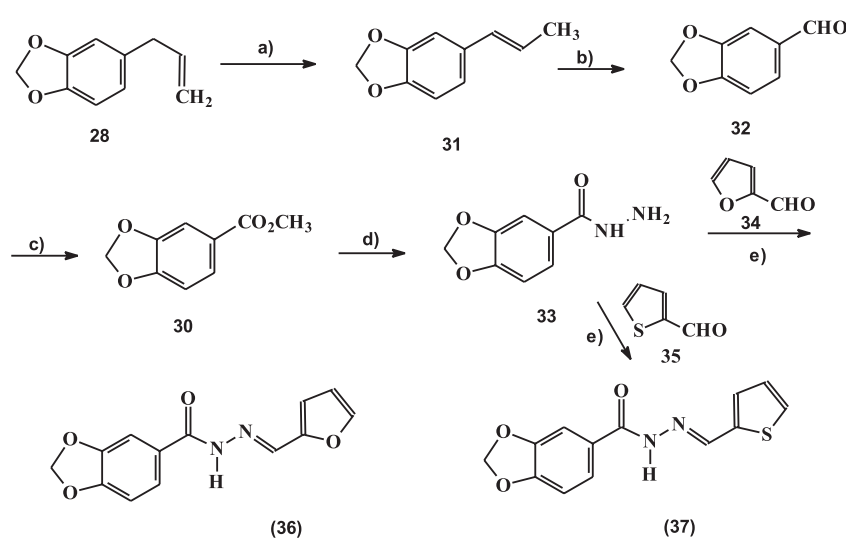

a) $\mathrm{KOH}$ aq. $3 \mathrm{~N}, \mathrm{tBuOH}$; b) $\mathrm{O}_{3}-\mathrm{O}_{2}, \mathrm{AcOH},-10^{\circ} \mathrm{C}, 4 \mathrm{~h} ; \mathrm{Zn}, \mathrm{AcOH}, 0{ }^{\circ} \mathrm{C}, 2 \mathrm{~h}$; c) $\mathrm{I}_{2}$,

$\mathrm{KOH}, \mathrm{MeOH}$; d) $\mathrm{N}_{2} \mathrm{H}_{4} \cdot \mathrm{H}_{2} \mathrm{O}, \mathrm{EtOH}$; e) Heteroaril- $\mathrm{CHO}$, EtOH, $\mathrm{HCl}$ (cat.).

Esquema 13

Uma vez sintetizados, em ótimos rendimentos globais, os novos derivados NAH, exemplificados por (36) e (37) (Esquema 13), foram submetidos aos ensaios farmacológicos relacionados aos efeitos cardiotônicos. Os novos compostos sintetizados (e.g. 36 e 37) foram avaliados em modelos farmacológicos relacionados ao metabolismo de $\mathrm{Ca}^{+2}$ e os resultados obtidos identificaram o composto tiofênico 37 (LASSBio-294) ${ }^{64}$ como o mais ativo na modulação do $\mathrm{Ca}^{+2}$ do $\mathrm{SR}^{65-67}$, confirmando as expectativas antecipadas quando do planejamento estrutural desta série de derivados NAH (27). Por exemplo, dentre os novos derivados NAH sintetizados como isósteros de LASSBio-294 ${ }^{52}$, os derivados 2-furânico (36, Esquema 13), benzênico (38) e 4-piridínico (39) não foram capazes de apresentar perfil de atividade similar ao LASSBio-294 (37) ${ }^{68}$, o que evidenciou a importância da presença do substituinte 2-tiofênico na insaturação imínica da função NAH, para a atividade moduladora do $\mathrm{Ca}^{+2}$. Outrossim, a inexpressiva atividade observada para o composto benzotiodiazol (40) ${ }^{69}$, isóstero do sistema benzodioxol de LASSBio-294 (37), indicou a importância desta sub-unidade aromática, inspirada no produto natural (28) empregado como matériaprima sintética, para a atividade farmacológica identificada em (37), reforçando o acerto dos critérios empregados na eleição dos substituintes das sub-unidades acila e iminíca dos novos compostos NAH. 


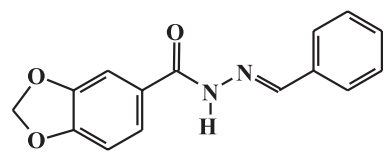

38

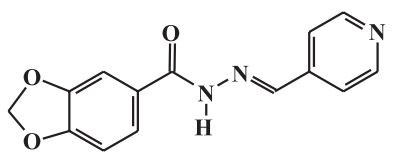

39<smiles>O=C(N/N=C/c1cccs1)c1ccc2nsnc2c1</smiles>

Considerando-se o envolvimento do $\mathrm{Ca}^{+2}$ do retículo sarcoplasmático na função muscular esquelética relacionada com a hipertermia maligna ${ }^{66}$ e distrofia muscular ${ }^{67}$, além do efeito cardioprotetor promovido pela modulação do $\mathrm{Ca}^{+2}$ citossólico ${ }^{67}$, direta ou indiretamente mediada por PDE's, o novo protótipo identificado, LASSBio-294 (37), foi então bioensaiado quanto às suas propriedades inotrópicas. Os resultados farmacológicos obtidos ${ }^{70-73}$ indicaram que LASSBio-294 (37) foi capaz de induzir intenso relaxamento, concentração-dependente, em anéis isolados e intactos de aorta de ratos com $\mathrm{IC}_{50} 74 \mu \mathrm{M}^{73}$. Ademais, este efeito foi abolido pela remoção do endotélio e não se alterou pela pré-inibição da $N$-óxido sintase (NOS), induzida por L-NAME (41), nem pela pré-inibição da cascata do ácido araquidônico, promovida pelo tratamento com concentração adequada de indometacina (15), conhecido inibidor da prostaglandina endoperóxido sintase (PGHS) ${ }^{73}$. Outrossim, o efeito de relaxamento observado com LASSBio-294 (37) permaneceu inalterado quando os anéis de aorta isolados de ratos foram pré-tratados com $\mathrm{K}^{+}$extracelular ${ }^{73}$. Estes resultados sugerem, fortemente, que o mecanismo de ação de (37), quanto aos seus efeitos inotrópicos, não é modulado pelo $N$-óxido (NO), nem pela cascata do ácido araquidônico ou por canais de $\mathrm{K}^{+}$podendo, efetivamente, estar relacionado com a inibição de PDE's.<smiles>CC(=O)C(N)CCCNC(=N)N[N+](=O)[O-]</smiles>

Face às atraentes propriedades identificadas para LASSBio-294 (37) os estudos farmacológicos foram ampliados, evidenciando que os efeitos inotrópicos positivos preliminarmente identificados neste novo derivado NAH eram $c$-GMP-dependentes, visto que podiam ser abolidos ou revertidos, na presença de inibidor seletivo de $c$ GMP, e.g. ODQ (42) ${ }^{73}$. Estes resultados reforçam seu envolvimento com o $\mathrm{Ca}^{+2}$ celular e com isoformas de PDE-c-GMP dependentes, presentes no músculo cardíaco $\left(\right.$ e.g. 2, 3 e 5) ${ }^{73}$. Cabe ressaltar que, embora a principal isoforma de PDE- $c$-GMP seletiva, presente no músculo cardíaco seja a PDE5, uma ação sobre a PDE3 não pode ser descartada, visto que essa isoforma reconhece como substrato ambos os nucleotídeos, $c$ AMP e $c$ GMP. De fato, a hipótese de inibição de PDE5 e 3 no mecanismo de ação de LASSBio-294 foi corroborada com a evidência experimental que identificou um perfil de atividade similar entre LASSBio-294 e o zaprinast (43) ${ }^{73}$, conhecido inibidor de PDE5 e PDE3, que possui índice de seletividade PDE5/ PDE3 de 50 e manifesta propriedades natriuréticas acessórias, aliás, empregado como protótipo no desenho estrutural do sildenafil (19) ${ }^{38}$.

Os bioensaios farmacológicos realizados com LASSBio-294 (37) indicaram, ainda, que esta substância apresentou tímidas propriedades antiinflamatórias, contrastando com outros derivados NAH sintetizados no laboratório ${ }^{14,59}$, pois em concentrações de $100 \mu \mathrm{M} / \mathrm{kg}$,<smiles>O=c1onc2cnc3ccccc3n12</smiles><smiles>CCCOc1ccccc1-c1nc2nn[nH]c2c(=O)[nH]1</smiles>

43

p.o., foi capaz de inibir o edema da pata de rato induzido por carragenina em apenas $20 \%$.

Curiosamente, quando LASSBio-294 (37) foi bioensaiado na inibição da agregação plaquetária $(100 \mu \mathrm{M})$ induzida por ácido araquidônico ou colágeno em plasma rico em plaquetas de coelho, inibiu em 100\% a agregação plaquetária. Outrossim, nesta mesma concentração, (37) não apresentou efeito anti-agregante quando a agregação plaquetária foi induzida por ADP, U-46619 e trombina ${ }^{74}$. Considerando-se que as principais isoformas de PDE presentes em plaquetas são as isoformas 3 e 5, os efeitos observados para o LASSBio-294 na inibição da agregação plaquetária são coerentes com aqueles obtidos em bioensaios com músculo liso e, em conjunto, permitem sugerir que este derivado NAH cardioativo esteja interferindo com a atividade destas isoformas de PDE. Contudo, a carência de inibidores específicos para muitas das isoformas de PDE's (e.g. PDE2), também presentes no músculo cardíaco, dificulta a identificação farmacológica das eventuais propriedades inibidoras seletivas de LASSBio-294 (37), não sendo possível descartar o envolvimento das isoformas 1 e 2 em seu mecanismo de ação.

O novo protótipo cardioativo (37) apresentou $\mathrm{ID}_{50} 8,2 \mu \mathrm{M} / \mathrm{kg}$ no modelo de analgesia das contorções induzidas por solução $0,2 \%$ de ácido acético (i.p. $)^{74}$, em camundongos, sem manifestar, entretanto, efeito em protocolos de ação central (e.g. "hot-plate", "tail-flick") ${ }^{74}$, eliminando a possibilidade de eventuais efeitos deletérios centrais.

Face ao atraente perfil farmacológico identificado para este autêntico protótipo cardioativo ${ }^{68-74}$, realizou-se uma investigação preliminar de seu perfil toxicológico, em ratos $(1000 \mu \mathrm{M} / \mathrm{kg}$, p.o. e i.p. $)$. Os resultados obtidos não evidenciaram nenhuma reação de toxicidade aguda (e.g. morte, letargia, perda de motilidade ou apetite $)^{74}$, credenciando este derivado NAH (37) como promissor protótipo, candidato a agente cardiotônico com propriedades seletivas no catabolismo do $\mathrm{Ca}^{+2}$ celular, ao nível do SR, atuando, provavelmente, como inibidor de PDE-c-GMP.

\section{CONCLUSÕES}

Neste trabalho ilustrou-se a importância da estratégia da simplificação molecular no planejamento racional de fármacos, a partir de produtos naturais ou sintéticos. A utilização desta estratégia da Química Medicinal permitiu a descoberta do novo derivado $\mathrm{N}$ acilidrazônico - LASSBio-294 (37) - que, face às suas importantes propriedades farmacológicas, foi objeto de recente depósito de patente $^{64}$. Este novo derivado NAH (37), de estrutura simples, descoberto pelo emprego da estratégia de simplificação molecular e sintetizado, em elevado rendimento global, a partir de produto natural brasileiro, abundante, acumula ações vasodilatadoras e potentes propriedades inotrópicas positivas, que o credenciam como autêntico composto-protótipo de novo fármaco cardiotônico para o tratamento da insuficiência cardíaca e/ou muscular (e.g. distrofia ou fadiga muscular), desprovido dos típicos efeitos deletérios dos glicosídeos $\operatorname{cardíacos}^{39}$, particularmente útil a pacientes de terceira idade.

Com base nas etapas hierárquicas do planejamento racional de novos fármacos, ilustrado na Figura 3, estudamos no laboratório, no momento, a otimização do protótipo LASSBio-294 e a elucidação de seu exato mecanismo farmacológico de ação. 


\section{AGRADECIMENTOS}

O autor agradece ao Prof. Dr. C. A. M. Fraga (LASSBio) e à Dra. L. M. Lima (LASSBio) pelos comentários, críticas e excelentes sugestões feitas durante a redação deste manuscrito. Agradece aos demais colegas do LASSBio, Profa. Dra. A. L. P. de Miranda e aos estudantes de pós-graduação P. C. Lima (DQO, IQ-UFRJ) e J. L. M. Tributino (DFBC, ICB-UFRJ). Agradece aos colegas do Departamento de Farmacologia Básica e Clínica do ICB-UFRJ, Dr. E. X. Albuquerque, Dr. R. T. Sudo, Dra. G. Zapata-Sudo, Dr. F. G. Nöel, Dra. C. L. M. Silva, Dra. Y. Aracava, Dr. N. G. Castro, Dr. P. A. Melo e Dr. W. M. Cintra e todos os seus colaboradores, pelo trabalho em equipe propiciado pelo Pronex, essencial na condução de projetos de Química Medicinal, caracteristicamente interdisciplinares, como o descrito neste trabalho. O autor agradece ao CNPq pela bolsa de produtividade e ao Pronex pelo apoio financeiro.

\section{REFERÊNCIAS}

1. Wermuth, C-G. Em The Practice of Medicinal Chemistry; Wermuth, CG., ed.; Academic Press: New York, 1996, p. 3; Ratti, E.; Trist, D.; Il Farmaco 2001, 56, 13.

2. Barreiro, E. J.; Fraga, C. A. M.; Química Medicinal: As Bases Moleculares da Ação dos Fármacos, Editora ArtMed Ltda.: Porto Alegre, 2001.

3. Black, J. W.; Duncan, W. A. M.; Durant, J. C.; Ganellin, C. R.; Parsons, M. E.; Nature 1972, 236, 385.

4. Ganellin, C. R.; J. Med. Chem. 1981, 24, 913; Ganellin, C. R. Em Chronicles of Drug Discovery; Bindra, J. S.; Lednicer, D., eds.; Wiley: New York, 1982, vol. 1, p. 1.

5. ref. 2, p. 163; Barreiro, E. J.; Rev. Bras. Farm. 1991, 72, 34

6. Patani, G. A.; LaVoie, E. J.; Chem. Rev. 1996, 96, 3147.

7. Bradshaw, J. Em Chronicles of Drug Discovery; Lednicer, D., ed.; ACS Books: Washington DC, 1993, vol. 3, p. 45; Ganellin, C. R. Em Medicinal Chemistry - The role of Organic Chemistry in Drug Research; Ganellin, C. R.; Roberts, S. M., eds.; Academic Press: Londres, 1993, p. 227; Gringauz, A. Em Introduction to Medicinal Chemistry: How Drugs Act and Why; Wiley: New York, 1997, p. 637.

8. Gund, P.; Maggiora, G.; Snyder, J. P. Em Guidebook on Molecular Modeling in Drug Design; Cohen, N. C., ed.; Academic Press: New York, 1996, p. 219.

9. Como evidência do desenvolvimento desta área temos o recente lançamento do Journal of Combinatorial Chemistry, pela American Chemical Society, Washington, EUA, http://pubs.acs.org/journals/jcchff/index.html, acessada em Janeiro 2002

10. Arlington, S.; Peakman, T.; DDT 2001, 6, 161. Vale ressaltar o lançamento em janeiro de 2002 do Journal of Proteome Research da American Chemical Society, Washington, EUA, http://pubs.acs.org/journals/jprobs/, acessada em Janeiro 2002.

11. Ganellin, C. R. Em Medicinal Chemistry Principles and Practice; King, F. D., ed.; RSC: London, 1994, p. 189; Gibbs, J.B.; Science 2000, 287, 1969.

12. Barreiro, E. J.; Silva, J. M. F.; Fraga, C. A. M.; Quim. Nova 1996, 19, 641 ; ref. 2 , p. 35

13. Wermuth, C-G.; J. Heterocycl. Chem. 1998, 35, 1091; Kubinyi, H.; Pharmazie 1995, 50, 647.

14. Para mais exemplos, veja: Barreiro, E. J.; Fraga, C. A. M.; Rodrigues, C. R.; Miranda, A. L. P.; Quim. Nova 2002, 25, 129.

15. Murcko, M. A.; Caron, P. R.; Charifson, P. S.; Annu. Rep. Med. Chem. 1999, 34, 297; Barreiro, E. J.; Albuquerque, M. G.; Sant'Anna, C. M. R.; Rodrigues, C. R.; de Alencastro, R. B.; Quim. Nova 1997, 20, 300.

16. Kubinyi, H.; Angew. Chem. Int., Ed. 1999, 38, 1824.

17. Ong, H. H.; Allen, R. C.; Annu. Rep. Med. Chem. 1988, 24, 295.

18. Setti, E. L.; Micetich, R.; Curr. Med. Chem. 1996, 3, 317.

19. Barreiro, E. J. Em Plantas Medicinais sob a Ótica da Química Medicinal Moderna; Yunes, R. A.; Calixto, J. B., eds.; Argos Editora Universitária, UNOESC: Chapecó, 2001, p. 250.

20. ref. 2, p. 99.

21. Murray, M. C.; Perkins, M. E.; Annu. Rep. Med. Chem. 1996, 31, 141.

22. Elion, G. B.; J. Med. Vir. 1993, Suppl. 1, 2.

23. Cushman, D. W.; Cheung, H.S.; Sabo, E. F.; Ondetti, M. A.; Biochemistry, 1977, 16, 5485.

24. Ferreira, S. H.; Bartelt, D. C.; Greene, L. J.; Biochemistry, 1970, 9, 2538.
25. Barreiro, E. J.; Fraga, C. A. M., Romeiro, N. C.; Lages, A. S.; Quim. Nova, 1998, 21, 761 .

26. McLeod, D. C.; Drugs Intell. Clin. Pharm. 1981, 15, 522.

27. Shen, T. Y.; Windholz, T. B.; Rosegay, A.; Witzel, B. E.; Wilson, A. N.; Willet, J. D.; Holtz, W. J.; Ellis, R. L.; Matzuk, A. R.; Lucas, S.; Stammer, C. H.; Holly, F. W.; Sarett, L. H.; Risley, E. A.; Nuss, G. W.; Winter, C. A.; J. Am. Chem. Soc. 1963, 85, 488; Shen, T. Y.; J. Med. Chem. 1981, 24 , 1.

28. Lin, J. H.; Ostovic, D.; Vacca, J. P. Em Integration of Pharmaceutical and Discovery and Development: Case Histories; Borchardt, R. T.; Freidinger, R. M.; Sawer, T. K.; Smith, P. L., eds.; Plenum Press: New York, 1998, p. 233.

29. Leung, D.; Abbenante, G.; Fairlie, D. P.; J. Med. Chem. 2000, 43, 305.

30. Tavel, J. A.; Exp. Opin. Invest. Drugs 2000, 9, 917.

31. Perry, M. J.; Higgs, G. A.; Curr. Opin. Chem. Biol. 1998, 2, 472.

32. Beavo,J. A.; Proc. Natl. Acad. Sci. U.S.A. 1999, 96, 7071; Fisher, D. A.; Smith, J. F.; Pillar, J. S.; St. Denis, S. H.; Cheng, J. B.; Biochem. Biophys. Res. Commun. 1998, 246, 570; Fisher, D. A.; Smith, J. F.; Pillar, J. S.; St. Denis, S. H.; Cheng, J. B.; J. Biol. Chem. 1998, 273, 15559; Beavo, J. A.; Reifsnyder, D. H.; Trends Pharmacol. Sci. 1990, 11, 150.

33. Soderling, S. H.; Beavo, J. A.; Curr. Opin. Cell Biol. 2000, 12, 174; Giembyez, M. A.; Drugs 2000, 59, 193.

34. Montana, J. G.; Dyke, H.J.; Annu. Rep. Med. Chem. 2001, 36, 41.

35. Hersperger, R.; Dawson, J.; Mueller, T.; Bioorg. Med. Chem. Lett. 2002, 12, 233.

36. Dal Piaz, V.; Giovannoni, M. P.; Eur. J. Med. Chem. 2000, 35, 463.

37. Burnouf, C.; Pruniaux, M-P.; Szilagyi, C. M.; Annu. Rep. Med. Chem. 1998, 33, 91

38. Lexchin, J.; Can. Med. Ass. J. 2001, 164, 1449; Langtry H. D.; Markham, A.; Drugs 1999, 57, 967; Maw, G. N.; Annu. Rep. Med. Chem. 1999, 34, 71; Terrett, N. K.; Bell, A. S.; Brown, D.; Ellis, P.; Bioorg. Med. Chem. Lett. 1996, 15, 1819.

39. Fraga, C. A. M.; Barreiro, E. J.; Quim. Nova 1996, 19, 182.

40. O principal representante desta classe é o captopril: Ondetti, M. A.; Rubin, B.; Cushman, D. W.; Science 1977, 196, 441; ref. 23.

41. O lozartan $\left(\right.$ Cozaar $\left.^{\circledR}\right)$ foi o primeiro fármaco desenvolvido, em 1995, para atuar ao nível dos receptores $\mathrm{AT}_{1}$, em posologia diária única: ref. 2, p. 97.

42. Diversos antagonistas de canais de $\mathrm{Ca}^{+2}$ foram descritos: ref 7, p. 492.

43. O principal representante é o prazozin desenvolvido pela Pfizer: Campbell, S.; Quim. Nova 1991, 14, 196.

44. Kagimoto, K.; Hagiwara, N.; Kasanuki, H.; Hosoda, S.; Br. J. Pharmacol. 1997, 121, 1549 .

45. Manganiello, V. C.; Taira, M.; Degerman, E.; Belfrage, P.; Cell. Signal. 1995, 7, 445 .

46. Diversos compostos diidro $(2 H)$-piridazinônicos apresentam propriedades cardiotônicas atuando ao nível da PDE3, inter-alia:: KF15232: Nomoto, Y.; Takai, H.; Ohno, T.; Nagashima, K.; Yao, K.; Kubo, K.; Ichimura, M.; Mihara, A.; Kase, H.; J. Med. Chem. 1996, 39, 297; Simendan: Pagel, P. S.; Haikala, H.; Pentikäinen, P. J.; Toivonen, M. L.; Nieminen, M. S.; Lehtonen, L.; Papp, J. G.; Warltier, D. C.; Cardiovasc. Drug. Rev. 1996, 14, 286.

47. Heinisch, G.; Kopelent-Frank, H.; Progr. Med. Chem. 1992, 29, 141;

48. Wakisaka, C.; Kitamura, N.; Ohta, T.; Kai, T.; Nakasato, Y.; Ito, S.; Fundam. Clin. Pharmacol. 2000, 14, 379.

49. Janis, R. A.; Triggle, D. J.; J. Med. Chem. 1983, 26, 775

50. Ukena, D.; Rentz, K.; Reiber, C.; Sybrecht, G. W.; Respiratory Medicine 1995, 89, 441 .

51. Mathew, L.; Katz, S. D.; Drugs \& Aging 1998, 12, 191.

52. Lima, P. C.; Lima, L. M.; da Silva, K. C. M.; Léda, P. H. O.; Miranda A. L. P.; Fraga, C. A. M.; Barreiro, E. J.; Eur. J. Med. Chem. 2000, 35, 187.

53. Santos, M. R. L.; Barreiro, E. J.; Bráz-Filho, R.; Miranda, A. L. P.; J. Braz. Chem. Soc. 1997, 8, 471 .

54. Jonas, R.; Lues, K. I.; Wurziger, H.; Bioorg. Med. Chem. Lett. 1994, 4, 2585; Verde, I.; Vandecasteele, G.; Lezoualc'h, F.; Fischmeister, R.; Br. J. Pharmacol. 1999, 127, 65.

55. Barreiro, E. J.; Fraga, C. A. M.; Quim. Nova 1999, 22, 744.

56. Inter-alia: Palnitkar, S. S.; Bin, B.; Jimenez, L. S.; Morimoto, H.; Williams, P. G.; Paul-Pletzer, K.; Parness, J.; J. Med. Chem. 1999, 42, 1872; Palnitkar, S. S.; Mickelson, J. R.; Louis, C. F.; Parness, J.; Biochem. J. 1997, 326, 847; Fruen, B. R.; Mickelson, J. R.; Louis, C. F.; J. Biol. Chem. 1997, 272, 26965; Nelson, T. E.; Lin, M.; Zapata-Sudo, G.; Sudo, R. T.; Anesthesiology 1996, 84, 1368; Parness, J.; Palnitkar, S. S.; J. Biol. Chem. 1995, 270, 18465; Ward, A.; Chaffman, M. O.; Sorkin, E. M.; Drugs 1986, 32, 130.

57. Hoffman, E. P.; Dressman, D.; Trends Pharmacol. Sci. 2001, 22, 465; Mickelson, J. R.; Louis, C. F.; Physiol. Rev. 1996, 76, 537; MacLennan, D. H.; Phillips, M. S.; Science 1992, 256, 789. 
58. Zhao, F. Y.; Li, P.; Chen, S. R. W.; Fruen, B. R.; J. Biol. Chem. 2001, 276, 13810.

59. Inter-alia: Figueiredo, J. M.; Camara, C. A.; Amarante, E. G.; Miranda, A. L. P.; Santos, F. M.; Rodrigues, C. R.; Fraga, C. A. M.; Barreiro, E. J.; Bioorg. Med. Chem. 2000, 8, 2243; Fraga, A. G. M.; Rodrigues, C. R.; Miranda, A. L. P.; Fraga, C. A. M.; Barreiro, E. J.; Eur. J. Pharm. Sci. 2000, 11, 285; Leite, L. F. C. C.; Ramos, M. N.; da Silva, J. B. P.; Fraga, C. A. M.; Barrreiro, E. J.; Il Farmaco 1999, 54, 747; Ribeiro, I. G.; Silva, K. C. M.; Parrini, S. C.; Miranda, A. L. P.; Fraga, C. A. M.; Barreiro, E.J.; Eur. J. Med. Chem. 1998, 33, 225; Pereira, A. S.; Violante, F. A.; Aquino Neto, F. R.; Cardoso, J. N.; Fraga, C. A. M.; Barreiro, E. J.; Anal. Lett. 1998, 31, 719; Gaston, M. A.; Dias, L. R. S.; Freitas, A. C. C.; Miranda, A. L. P.; Barreiro, E. J.; Pharm. Acta Helv. 1996, 71, 213.

60. Coelho, F. A. S.; Costa, P. R. R; de Farias, F. M. C.; Barreiro, E. J.; Quim. Nova 1984, 7, 113.

61. Lima, M. E. F.; Barreiro, E. J.; J. Pharm. Sci. 1992, 81, 1219.

62. Barreira, E. S.; Monte, F. J. Q.; Quim. Nova 1995, 18, 245.

63. Yamada, S.; Morizono, D.; Yamamoto, K.; Tetrahedron Lett. 1992, 33, 4329.

64. Albuquerque, E. X.; Barreiro, E. J.; Sudo, R. T.; US pat. Provisorial Number 60-525,352 1999.

65. Zucchi, R.; Ronca, F.; Ronca-Testoni, S.; Pharmacol. Ther. 2001, 89, 47.

66. Berchtold, M. W.; Brinkmeier, H.; Muntener, M.; Physiol. Rev. 2000, 80, 1215 .
67. Ruegg, U. T.; Gillis, J-M.; Trends Pharmacol. Sci. 1999, 20, 351.

68. Zapata-Sudo, G.; Sudo, R. T.; Aguiar, M. I. S.; Lima, P. C.; Fraga, C. A. M.; Barreiro, E. J.; Resumos da XIII Reunião Anual da Federação de Sociedades de Biologia Experimental, Caxambú, Brasil, 1998.

69. Barreiro, E. J.; Sudo, R. T.; Zapata-Sudo, G.; Fraga, C. A. M.; Ferreira, M. C. B.; trabalho não publicado.

70. Sudo, R. T.; Zapata-Sudo, G.; Barreiro, E. J.; Br. J. Pharmacol. 2001, 134, 603.

71. Gonzalez-Serratos, H.; Chang, R.; Pereira, E. F. R.; Castro, N. G.; Aracava, Y.; Melo, P. A.; Lima, P. C.; Fraga, C. A. M.; Barreiro E. J.; Albuquerque, E. X.; J. Pharmacol. Exp. Ther. 2001, 299, 558.

72. Silva, C. L. M.; Nöel, F.; Barreiro, E. J.; Fraga, C. A. M.; Lima, P. C.; Resumos da XIV Reunião Anual da Federação de Sociedades de Biologia Experimental, Caxambú, Brasil, 1999; Silva, C. L. M.; Nöel, F.; Fraga, C. A. M.; Barreiro, E. J.; Abstracts of XVI Latinamerican Congress of Pharmacology, Águas de Lindóia, Brasil, 2000.

73. Silva, C. L. M.; Noël, F.; Barreiro, E. J.; Br. J. Pharmacol. 2002, 135 , 293.

74. Miranda, A. L. P.; Lima, P. C.; Tributino, J. L. M.; Melo, P. A.; Fernandes, P. D.; Cintra, W. M.; Fraga, C. A. M.; Barreiro, E. J.; trabalho não publicado. 